\title{
WANDERING DOMAINS FOR DIFFEOMORPHISMS OF THE $k$-TORUS: A REMARK ON A THEOREM BY NORTON AND SULLIVAN
}

\author{
Andrés Navas \\ Univ. de Santiago de Chile, Fac. de Ciencia, Dpto de Matemática y C.C. \\ Alameda 3363, Estación Central, Santiago, Chile; andres.navas@usach.cl
}

\begin{abstract}
We show that there is no $C^{k+1}$ diffeomorphism of the $k$-torus which is semiconjugate to a minimal translation and has a wandering domain all of whose iterates are Euclidean balls.
\end{abstract}

\section{Introduction}

Answering (in the negative) a question raised by Poincaré [17], Denjoy proved one of his most famous theorems [4], namely the action of every $C^{2}$ (orientation preserving) diffeomorphism of the circle with irrational rotation number is minimal. This result can be considered as one of the the starting points of the theory of differentiable dynamics, and many generalizations have been proposed in the context of one-dimensional dynamics (see, for example, $[3,5,6,19,20,25]$ ). The search for higher dimensional analogues of the Denjoy Theorem is a natural problem that has attracted some interest in recent years. Although there is some partial evidence in the positive direction, no definitive result is known.

Let us be more precise. As is well known, the $C^{2}$ (or $C^{1+\text { Lip }}$ ) hypothesis is necessary in the statement of the Denjoy Theorem. Indeed, Denjoy himself gave $C^{1}$ counter-examples for his result. (It should be noticed that the first $C^{1}$ counterexamples were constructed by Bohl in [2].) These examples were improved by Herman in [8] up to the class $C^{2-\varepsilon}$ for every $\varepsilon>0$ (se also [22]), thus showing that the Denjoy Theorem is sharp in the Hölder scale. The blowing-up method of Denjoy and Herman is classical and direct. However, circle diffeomorphisms with similar properties can be obtained as holonomy maps along stable manifolds of Anosov diffeomorphisms of the 2-torus. McSwiggen extended this construction in $[11,12]$ to higher dimensions and showed that, for each $k \geq 2$, there exists a dense family $\left(\theta_{1}, \ldots, \theta_{k}\right)$ of $\mathbf{Q} / \mathbf{Z}$-independent numbers in $\mathbf{R} / \mathbf{Z}$ such that, for any $\varepsilon>0$, there is a $C^{k+1-\varepsilon}$ diffeomorphism of the $k$-torus that has a wandering (topological) disk and is semiconjugate to the translation by $\left(\theta_{1}, \ldots, \theta_{k}\right)$. Unfortunately, the family of rotation vectors which appear in McSwiggen's constructions is countable (they are all algebraic, and therefore Diophantine), and it is unclear whether similar examples do exist for any translation vector with the above properties.

McSwiggen's examples show that the natural differentiability where we should look for an analogue of the Denjoy Theorem on the $k$-torus is $C^{k+1}$. This is confirmed by the fact that, by a straightforward application of the KAM Theory, if $f$ is a small $C^{k+1+\varepsilon}$ perturbation of an irrational Diophantine translation of the $k$-torus, then $f$ is $C^{k}$ conjugate to it [7].

https://doi.org/10.5186/aasfm.2018.4314

2010 Mathematics Subject Classification: Primary 30L10, 37C05, 37E30.

Key words: Denjoy's theorem, quasi-conformal map, distortion. 
In the one-dimensional case, the blowing-up procedure for the construction of counter-examples is necessarily "conformal": one replaces points along orbits by intervals. In higher dimensions, one should replace points by continua, and the case where these continua are not topological disks is interesting by itself: see, for instance, $[1,18]$. (We should point out that, in this case, perhaps a Denjoy type theorem holds in regularity smaller than $C^{k+1}$.) In the case of wandering topological disks, several partial results are known in dimension 2 (see for example [14, 15, 16]). For instance, in [15], Norton and Sullivan show that it is impossible for a $C^{3}$ diffeomorphism of the 2-torus to be semiconjugate and non-conjugate to a minimal translation, provided that the preimages of points have some "uniform" conformal geometry along the orbits.

The aim of this Note is to show how a simple modification of some of the ideas of [15] allows proving similar results for $C^{k+1}$ diffeomorphisms of the $k$-torus. It should be emphasized that these do not follow from Norton-Sullivan's arguments, as these strongly rely on the Morrey-Bojarski-Ahlfors-Bers integration theorem, which is no longer available in higher dimensions. In particular, one of the key arguments of [15] uses Sullivan's integrability theorem, which states that every uniformly quasiconformal group of homeomorphisms of a surface is quasiconformally conjugate to a group of conformal maps [21], and which is known to be false in dimension greater than two [23].

For the sake of concreteness, we only prove the following theorem, which is somewhat the core of [15].

Theorem. Let $k \geq 2$, and let $f$ be a diffeomorphism of the $k$-torus that is semiconjugate to a minimal translation without being conjugate to it. If the preimage by the semiconjugacy of each point is either a point or an Euclidean ball, then $f$ cannot be of class $C^{k+1}$.

Note that this result is still true in dimension one (where it follows from the classical Denjoy Theorem), but our arguments only work in higher dimensions (see, however, [13, Exercise 3.1.4], which is somewhat related to our arguments here).

We should stress that we do not know whether the $C^{k+1}$ regularity hypothesis is actually needed for our Theorem (assuming that the wandering domains are Euclidean balls). Indeed, in McSwiggen's examples, the wandering domains have a very irregular geometry, and there is even no uniform bound for the diameter of their lifts. See also [10] for a recent interesting result concerning topological entropy of this kind of maps.

Combining the methods of this Note with those of [9], one can show that if $f_{i}$, $i \in\{1, \ldots, d\}$, are respectively $C^{1+k_{i}}$ diffeomorphisms of the $k$-torus that are semiconjugate to minimal translations and whose translation vectors are independent over $\mathbf{Q} / \mathbf{Z}$, then one has $k_{1}+\ldots+k_{d} \leq k$ provided the $f_{i}$ 's commute and the preimages of points by the (simultaneous) conjugacy to translations are either points or Euclidean balls. (The constants $k_{i}$ 's are supposed to be positive but not necessarily integer numbers). Actually, the same statement holds without the hypothesis that the $f_{i}$ commute but asking for the commutativity of their permutation action along an orbit of balls arising from the blowing-up procedure.

Acknowledgments. This note circulated as a manuscript more than ten years ago. I'm indebted to J. Kiwi, V. Kleptsyn and M. Ponce for their comments at that time, to all my colleagues who asked me to make this available (despite no progress has been made since then), and to J. Bochi for his insight concerning Lemma 1 below. 
The preparation of this text was funded by the CONICYT Project 1415 "Geometría en La Frontera" and the Fondecyt grant 11060541. Besides, this was supported by the LABEX MILYON (ANR-10-LABX-0070) of Université de Lyon, within the program "Investissements d'Avenir" (ANR-11-IDEX-0007) operated by the French National Research Agency (ANR). Finally, I whish to thank the Institute of Pure Mathematics of Teheran (Iran) for the hospitality during this task, and M. Nassiri for his invitation.

\section{Proof of the Theorem}

Let $\operatorname{Conf}(k) \sim \operatorname{GL}(k, \mathbf{R}) /(\mathrm{SO}(k, \mathbf{R}) \times \mathbf{R})$ denote the space of conformal structures on $\mathbf{R}^{k}$. This is a simply-connected space that carries a nonpositively curved metric which is invariant under the $\operatorname{GL}(k, \mathbf{R})$-action given by $A \cdot[B]:=[A B]$. In particular, the distance function dist $_{k}$ on it is smooth. For simplicity, we denote $\sigma_{0}:=[I d]$.

Lemma 1. If $f$ is a $C^{k}$ diffeomorphism satisfying the hypothesis of the Theorem, then there exists a constant $M$ such that $\operatorname{dist}_{k}\left(\left[D f^{n}(x)\right], \sigma_{0}\right) \leq M$ holds for all $x \in \mathbf{T}^{k}$ and all $n \geq 1$.

Proof. Let $\varphi$ denote the semiconjugacy of $f$ to the corresponding translation. Then

$$
\Gamma:=\mathbf{T}^{k} \backslash \bigcup \text { interior }\left(\left\{\varphi^{-1}(x): x \in \mathbf{T}^{k}\right\}\right)
$$

is a connected, nonwhere dense, minimal invariant set for $f$ (see [16]). Moreover, since all the wandering topological balls are Euclidean, $[D f(x)]$ is identically equal to $\sigma_{0}$ on $\Gamma$. Furthermore, as dist $_{k}$ is smooth, the derivatives of the function $x \mapsto$ $\operatorname{dist}_{k}\left([D f(x)], \sigma_{0}\right)$ vanish up to order $k$. By a successive application of the Mean Value Theorem, this implies that there exists a constant $C$ such that, if $x$ belongs to the interior of a ball $B_{x}$ that collapses to a single point under $\varphi$, then

$$
\operatorname{dist}_{k}\left([D f(x)], \sigma_{0}\right) \leq C \ell(x)^{k},
$$

where $\ell(x)$ is the half of the length of the shortest chord of $B_{x}$ through $x$. Since $\ell(x) \leq \operatorname{radius}\left(B_{x}\right)$, this shows that

$$
\operatorname{dist}_{k}\left([D f(x)], \sigma_{0}\right) \leq M \operatorname{vol}\left(B_{x}\right)
$$

for a certain constant $M$. This yields, for each $n \geq 1$ and every $x \notin \Gamma$,

$$
\begin{aligned}
\operatorname{dist}_{k}\left(\left[D f^{n}(x)\right], \sigma_{0}\right) & =\operatorname{dist}_{k}\left(D f^{n}(x) \cdot \sigma_{0}, \sigma_{0}\right) \\
& \leq \sum_{i=0}^{n-1} \operatorname{dist}_{k}\left(D f^{i+1}\left(f^{n-i-1}(x)\right) \cdot \sigma_{0}, D f^{i}\left(f^{n-i}(x)\right) \cdot \sigma_{0}\right) \\
& =\sum_{i=0}^{n-1} \operatorname{dist}_{k}\left(D f^{i}\left(f^{n-i}(x)\right)\right) \cdot\left[D f\left(f^{n-i-1}(x)\right], D f^{i}\left(f^{n-i}(x)\right) \cdot \sigma_{0}\right) \\
& =\sum_{i=0}^{n-1} \operatorname{dist}_{k}\left(\left[D f\left(f^{n-i-1}(x)\right)\right], \sigma_{0}\right) \\
& \leq \sum_{i=0}^{n-1} M \operatorname{vol}\left(B_{f^{n-i-1}(x)}\right) \\
& \leq M
\end{aligned}
$$

where the last inequality holds because the balls $B_{f^{j}(x)}$ are two-by-two disjoint. Since $\Gamma$ is nonwhere dense in $\mathbf{T}^{k}$, the estimate above holds for every $x \in \mathbf{T}^{k}$. 
Remark. The dilatation of an invertible linear map $A: \mathbf{R}^{k} \rightarrow \mathbf{R}^{k}$ is defined as

$$
\operatorname{dil}(A):=\frac{\max _{\|v\|=1}\|A(v)\|}{\min _{\|w\|=1}\|A(w)\|} .
$$

This induces a function on $\operatorname{Conf}(n)$ that measures the degree of non-conformality of matrices. In the 2-dimensional case, this is a smooth function, as is shown by the well-known formula

$$
\operatorname{dil}(A)=\frac{1+\|\mu\|}{1-\|\mu\|}=\exp \left(\operatorname{dist}_{\mathrm{hyp}}(0, \mu)\right),
$$

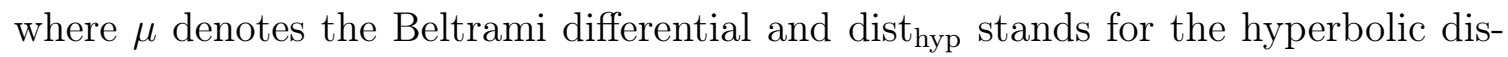
tance on the Poincaré disk. However, in higher dimension, this function is no longer smooth (yet it is locally Lipschitz). This is why, unlike [15], we do not deal with the function dil, and we directly consider the function dist $_{k}$.

Lemma 2. For each $M>0$ there exist $\lambda>1$ and $\lambda^{\prime}>1$ with the following property: if $g$ is a diffeomorphism of $\mathbf{R}^{k}$ that commutes with the translations by vectors in $\mathbf{Z}^{k}$ and such that $\operatorname{dist}_{k}\left([D g(x)], \sigma_{0}\right) \leq M$ for all $x \in \mathbf{R}^{k}$ and $g\left(B\left(x_{0}, \alpha\right)\right)=$ $B\left(y_{0}, \beta\right)$ for some $x_{0}, y_{0}$ in $\mathbf{R}^{k}$ and some positive numbers $\alpha, \beta$, then

$$
g\left(B\left(x_{0}, \lambda \alpha\right)\right) \subset B\left(y_{0}, \lambda^{\prime} \beta\right) .
$$

Proof. This follows directly from the equicontinuity of the family of restrictions to $B\left(x_{0}, 2 \alpha\right)$ of the maps $g$ satisfying the properties above; see $[24, \S 19]$.

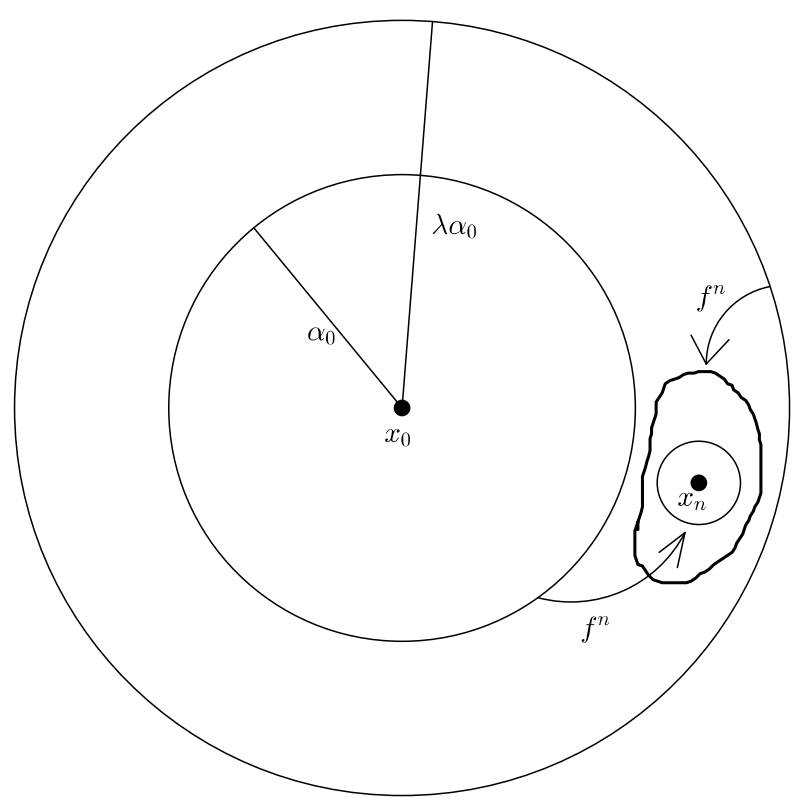

To complete the proof of the Theorem, we will use an argument that, in the onedimensional context, goes back to Schwartz [20]. Fix a wandering ball $B=B\left(x_{0}, \alpha_{0}\right)$, and denote by $x_{n}$ (resp. $\alpha_{n}$ ) the center (resp. the radius) of $f^{n}(B)$. Clearly, $\alpha_{n}$ goes to zero as $n$ goes to infinite, and for each $\varepsilon>0$ one has $f^{n_{k}}(B) \subset B\left(x_{0}, \alpha_{0}+\varepsilon\right)$ for an increasing sequence $\left(n_{k}\right)$ of positive numbers. Fix such an $n=n_{k}$ so that

$$
\alpha_{n}<\frac{(\lambda-1) \alpha_{0}}{2 \lambda^{\prime}} \text { and } \operatorname{dist}\left(x_{0}, x_{n}\right)<\alpha_{0}+\left(\frac{\lambda-1}{2}\right) \alpha_{0} \text {. }
$$


We can apply Lemma 2 to any covering map $\tilde{f}$ of $f$, thus obtaining

$$
\tilde{f}^{n_{k}}\left(B\left(\tilde{x}_{0}, \lambda \alpha_{0}\right)\right) \subset B\left(\tilde{x}_{n}, \lambda^{\prime} \alpha_{n}\right) \subset B\left(\tilde{x}_{0}, \alpha_{0}+\left(\frac{\lambda-1}{2}\right) \alpha_{0}+\lambda^{\prime} \alpha_{n}\right) \subset B\left(\tilde{x}_{0}, \lambda \alpha_{0}\right) .
$$

Therefore, $f^{n}\left(\overline{B\left(x_{0}, \lambda \alpha_{0}\right)}\right) \subset \overline{B\left(x_{0}, \lambda \alpha_{0}\right)}$, and by Brouwer's fixed point theorem, $f^{n}$ has a fixed point in $\overline{B\left(x_{0}, \lambda \alpha_{0}\right)}$. However, this is absurd, since $f$ is semiconjugate to a minimal (and therefore periodic-point free) torus translation. This contradiction completes the proof.

\section{References}

[1] Béguin, F., S. Crovisier, and F. Le Roux: Construction of curious minimal uniquely ergodic homeomorphisms on manifolds: the Denjoy-Rees technique. - Ann. Sci. Éc. Norm. Supér. (4) 40, 2007, 251-308.

[2] Bohl, P.: Uber die hinsichtlich der unabhängigen variabeln periodische. - Acta Math. 40, $1916,321-336$.

[3] De Melo, W., and S. Van Strien: A structure theorem in one dimensional dynamics. Ann. of Math. 129, 1989, 519-546.

[4] Denjoy, A.: Sur les courbes définies par des équations différentielles à la surface du tore. - J. Math. Pure Appl. 11, 1932, 333-375.

[5] Deroin, B., V. Kleptsyn, and A. Navas: Sur la dynamique unidimensionelle en régularité intermédiaire. - Acta Math. 199, 2007, 199-262.

[6] Ghys, É.: Transformations holomorphes au voisinage d'une courbe de Jordan. - C. R. Acad. Sci. Paris 298, 1984, 385-388.

[7] Herman, M.: Sur les courbes invariantes par les difféomorphismes de l'anneau. Volumes 1 and 2. - Astérisque 103-104 and 144, Soc. Math. France, 1983 and 1986.

[8] Herman, M.: Sur la conjugaison différentiable des difféomorphismes du cercle à des rotations. - Publ. Math. Inst. Hautes Études Sci. 49, 1979, 5-234.

[9] Kleptsyn, V., and A. Navas: A Denjoy type theorem for commuting circle diffeomorphisms with different Hölder differentiability classes. - Moscow Math. Journal 8, 2008, 477-492.

[10] Kwakkel, F., and V. Markovic: Topological entropy and diffeomorphisms of surfaces with wandering domains. - Ann. Acad. Sci. Fenn. Math. 35:2, 2010, 503-513.

[11] McSwiggen, P.: Diffeomorphisms of the torus with wandering domains. - Proc. Amer. Math. Soc. 117, 1993, 1175-1186.

[12] McSwiggen, P.: Diffeomorphisms of the $k$-torus with wandering domains. - Ergodig Theory Dynam. Systems 15, 1995, 1189-1205.

[13] Navas, A.: Groups of circle diffeomorphisms. - Chicago Lectures in Mathematics, 2011.

[14] Norton, A.: An area approach to wandering domains. - Ergodic Theory Dynam. Systems 11, 1991, 455-467.

[15] Norton, A., and D. Sullivan: Wandering domains and invariant conformal structures for mappings of the 2-torus. - Ann. Acad. Sci. Fenn. Math. 21, 1996, 51-68.

[16] Norton, A., and J. Welling: Conformal irregularity for diffeomorphisms of the 2-torus. Rocky Mountain J. Math. 24, 1994, 651-671.

[17] Poincaré, H.: Mémoire sur les courbes définies par une équation différentielle III. - J. Math. Pure Appl. 1, 1885, 167-244.

[18] Rees, M.: A minimal positive entropy homeomorphism of the 2-torus. - J. London Math. Soc. 23, 1981, 537-550.

[19] Sacksteder, R.: Foliations and pseudogroups. - Amer. J. Math. 87, 1965, 79-102. 
[20] Schwartz, A.: A generalization of Poincaré-Bendixon theorem to closed two dimensional manifolds. - Amer. J. Math. 85, 1963, 453-458.

[21] Sullivan, D.: Riemann surfaces and related topics. - In: Proc. Stony Brook Conf., Stony Brook, N.Y., 1978, Ann. of Math. Stud. 97, Princeton Univ. Press, Princeton, N.J., 1981, 465-496.

[22] TsuBoi, T.: Homological and dynamical study on certain groups of Lipschitz homeomorphisms of the circle. - J. Math. Soc. Japan 47, 1995, 1-30.

[23] Tukia, P.: A quasiconformal group not isomorphic to a Möbius group. - Ann. Acad. Sci. Fenn. Ser. A I Math. 6:1, 1981, 149-160.

[24] VÄIsÄLÄ, J.: Lectures on n-dimensional quasiconformal mappings. - Lecture Notes in Math. $229,1971$.

[25] Yoccoz, J.-C.: Il n'y a pas de contre-exemple de Denjoy analytique. - C. R. Acad. Sci. Paris $298,1984,141-144$.

Received 5 February 2017 • Accepted 8 September 2017 